Original Article

\title{
Does treadmill walking with near-infrared light applied to the abdominal area reduce local adiposity and body weight?
}

\author{
Sungwoon Kim, PhD ${ }^{1)}$, Yujin Kim, MS ${ }^{1)}$, Gyusung Lee, PhD ${ }^{1)}$, Jingu Kim, PhD $^{1)^{*}}$ \\ 1) Department of Physical Education, Kyungpook National University: 80 Daehakro, Bukgu, Daegu \\ 702-701, Republic of Korea
}

\begin{abstract}
Purpose] The purpose of this study was to determine the effect on local fat deposition and body weight of wearing a near-infrared light belt around the abdomen. [Subjects and Methods] Twenty-eight obese female subjects participated in this experiment. For measurement of body composition, an impedance-style body fat analyzer was used. The experimental group performed treadmill walking 3 times per week for 12 weeks while wearing an activated near-infrared belt around the abdomen. The near-infrared belt was composed of light-emitting diodes having wavelengths of $630 \mathrm{~nm}, 830 \mathrm{~nm}, 880 \mathrm{~nm}$, and $956 \mathrm{~nm}$. [Results] The analysis of abdominal circumference indicated that the abdominal circumference of the experimental group was reduced in post-test measurements compared to pre-test. [Conclusion] This investigation showed significant reductions in abdominal circumference, abdominal fat percentage, fat mass, and body-mass index for the experimental compared to the control group, suggesting that changes in body composition can be enhanced when near-infrared radiation is applied to the abdomen during walking. Key words: Body composition, Blood test, Near-infrared light
\end{abstract}

(This article was submitted May 29, 2017, and was accepted Jul. 1, 2017)

\section{INTRODUCTION}

Obesity is emerging as a serious social problem in modern society. Obesity causes various diseases by excessive accumulation of adipose tissue. Most notably, as the obesity level increases, the risk factors for and morbidity of cardiovascular disease increase ${ }^{1)}$. Obesity has also been identified as a major health risk factor, increasing the risk not only for hypertension, type 2 diabetes, dyslipidemia, sleep apnea, liver dyslipidemia, and endocrine disorders, but also affecting the individual's mental health and well-being 2,3 .

Until now, most studies on the treatment of obesity have focused on solving the imbalance of metabolic function and the approach is usually through dietary and exercise therapies ${ }^{4}$. However, even if exercise with a relatively low risk of side effects is performed, it can still lead to side effects such as ligament injuries and arthritis, depending on individual characteristics and athletic abilities ${ }^{5}$. Recently, there has been increasing interest in attempting to treat obesity using non-invasive near-infrared radiation. Near-infrared radiation (NIR) is light existing between visible-spectrum rays and mid-infrared rays, longer than a visible ray and shorter than a mid-infrared ray, with a wavelength of 0.72 to $1.6 \mu \mathrm{a}$.

In a prior study, obese adolescents, wearing an abdominal band that radiated near-infrared rays, performed aerobic exercise for 50 minutes 3 times a week for 4 weeks. It was found that body-mass index (BMI), waist circumference, and fat level were significantly more decreased in the group who were treated with both aerobic exercise and NIR, compared to those who only had aerobic exercise ${ }^{6}$. In addition, Mecrea et al. investigated the effect of water-filtered infrared-A (wIRA) on local fat reduction during moderate bicycle ergometer endurance exercise, and found greater decrease in the circumferences of waist,

*Corresponding author. Jingu Kim (E-mail: jigkim@knu.ac.kr)

(C2017 The Society of Physical Therapy Science. Published by IPEC Inc.

(c) $(-)$ This is an open-access article distributed under the terms of the Creative Commons Attribution Non-Commercial No DerivaBY NC ND tives (by-nc-nd) License. (CC-BY-NC-ND 4.0: http://creativecommons.org/licenses/by-nc-nd/4.0/) 
hip, and both thighs in the wIRA group relative to the control group ${ }^{7)}$. This study used the method of distant irradiation while subjects were using the ergometer. While suggesting the positive influence of the NIR on reducing obesity, these studies have a limitation of low practicality. Therefore, the present study aims to investigate changes in abdominal fat, body fat and BMI when middle-aged females with a high abdominal obesity rate performed treadmill exercise wearing a band equipped with 630-nm, 830-nm, 880-nm, and 956-nm near-infrared LEDs.

\section{SUBJECTS AND METHODS}

Twenty-eight female participants whose age ranged from 50 to 65 with a mean age of 56.75 years, mean height of $156.26 \mathrm{~cm}$, (Standard Deviation (SD), 2.82), mean weight of $74.67 \mathrm{~kg}$ (SD, 7.46), and BMI over $29 \mathrm{~kg} / \mathrm{m}$ ), were recruited from several fitness centers in Daegu, South Korea. Participants were assigned to either the exercise group ( $\mathrm{n}=14)$ or the control group $(n=14)$ using a purposive sampling method. All participants gave informed consent prior to the experiment in accordance with the ethical standard of the Declaration of Helsinki.

Body fat percentage, abdominal fat percentage, and BMI were measured using an Inbody 3.0 (bioelectrical impedance fatness analyzer GIF-891DH, Inbody, Kilwoo, Korea). Abdominal obesity of the participants was identified using the waist/ hip circumference ratio of $\geq 80 \mathrm{~cm}$, which was suggested by the WHO West Pacific Region in 2000. The thickness of fat of the participants was measured with a skinfold caliper (Idass, England) at three areas including at thigh, finger, and upper arm.

To measure total cholesterol, high-density lipoprotein (HDL) cholesterol, low-density lipoprotein (LDL) cholesterol, and triglycerides, nurses performed blood sampling for all participants. On the day of the experiment, $5 \mathrm{ml}$ of venous blood was collected at the cubital fossa from each participant after more than 30 minutes' rest. Samples were analyzed at Daegu Clinical Testing Center.

This study was carried out with a walking program at $60 \%$ of maximum heart rate (HRmax 60\%), performed at Kyungpook National University's fitness center 3 times a week for 12 weeks. As the experimental treatment, walking was performed on a treadmill using the wireless heart rate monitor, X-trainer (Polar Ambulatory heart-rate monitor, Polar, Port Washington, NY, USA). In order to maintain the target heart rate (HRmax 60\%), participants went through a one-week adaptation process supervised by the exercise prescriber, so that they would understand and learn correct posture and ergonomic movements for walking. The 12 -week walking exercise program was devised based on the study by Hiatt et $\mathrm{l}^{8}$ ). A pedometer (YAMASA, EM-180, Japan) was distributed to each participant for precise recording of the amount of walking exercise each day. The experimental group performed treadmill walking while wearing an activated near-infrared belt around the abdomen. The near-infrared belt was composed of light-emitting diodes having wavelengths of $630 \mathrm{~nm}, 830 \mathrm{~nm}, 880 \mathrm{~nm}$, and $956 \mathrm{~nm}$.

The Control group wore the abdominal belt, but it was inactivated and hence free of near-infrared rays; this group performed the same amount of treadmill walking as the experimental group. A 2 (group) $\times 2$ (pre- and post-) ANOVA with repeated measures on the last factor was carried out. Dependent variables were body composition factors (abdominal circumference, abdominal fat percentage, BMI, fat mass, body fat) and blood components (total cholesterol, HDL cholesterol, LDL cholesterol, triglycerides). Post hoc analysis using Bonferroni was performed to determine where the significant differences were located. Statistical data were analyzed using the SPSS 21.0 program, and the statistical significance level was set at $\alpha=0.05$.

\section{RESULTS}

The analysis of abdominal circumference indicated that the abdominal circumference of the experimental group was reduced in post-exercise measurements compared to pre-exercise $(\mathrm{p}<0.001)$. In the analysis of abdominal fat ratio, the interaction between group and measurement time (pre- or post-exercise) was also significant $(F(1,26)=8.67, p<0.01)$. Post-hoc test results showed that the abdominal fat rate in the experimental group was reduced in post-test measurements compared to pre-exercise $(\mathrm{p}<0.001)$. The analysis of BMI revealed that BMI in the experimental group was reduced in post-exercise compared to pre-exercise $(\mathrm{p}<0.001)$. In the analysis of fat mass, the interaction between group and measurement time was also significant $(\mathrm{F}(1,26)=32.16, \mathrm{p}<0.001)$. Post-hoc test results showed that fat mass in the experimental group was reduced in post-exercise measurements compared to pre-exercise $(\mathrm{p}<0.001)$. Body fat percentage analysis showed that fat mass percentage in the experimental group was reduced in post-exercise measurements compared to pre-exercise $(\mathrm{p}<0.001)($ Table 1$)$. Analysis of total cholesterol, HDL cholesterol, LDL cholesterol, and neutral fat (triglyceride) showed no group difference or significant interaction before and after the treadmill walking.

\section{DISCUSSION}

The purpose of this study was to determine the effects of treadmill walking with a near-infrared light-emitting belt around the abdomen on the reduction of local body fat and BMI index. As a result of analyzing body changes (abdominal circumference, abdominal fat percentage, body fat mass, body fat percentage, body-mass index), the experimental group who performed walking with the near-infrared belt showed significant reduction in abdominal circumference, abdominal 
Table 1. Mean and standard deviation of sub-factors of body composition by group and measurement time (pre- or post-exercise)

\begin{tabular}{llcc}
\hline \multirow{2}{*}{ Group } & Sub-factors & Pre-exercise & Post-exercise \\
\cline { 3 - 4 } & Abdominal circumference $(\mathrm{cm})$ & Mean $\pm \mathrm{SD}$ & Mean $\pm \mathrm{SD}$ \\
\hline \multirow{2}{*}{$\begin{array}{l}\text { Experimental } \\
\text { group }\end{array}$} & Abdominal fat rate $(\mathrm{WHR}, \mathrm{cm})$ & 104.48 & $99.95^{*}$ \\
& BMI $\left(\mathrm{kg} / \mathrm{m}^{2}\right)$ & 30.94 & $0.981^{*}$ \\
& Fat mass $(\mathrm{kg})$ & 32.98 & $29.61^{*}$ \\
& Body fat percentage $(\%)$ & 42.79 & $29.21^{*}$ \\
& Abdominal circumference $(\mathrm{cm})$ & 100.56 & 39.50 \\
Control group & 1.00 & 0.13 \\
$(\mathrm{n}=14)$ & Abdominal fat rate $(\mathrm{WHR}, \mathrm{cm})$ & 30.32 & 29.79 \\
& BMI $\left(\mathrm{kg} / \mathrm{m}^{2}\right)$ & 30.18 & 29.26 \\
& Fat mass $(\mathrm{kg})$ & 41.03 & 40.48 \\
\hline
\end{tabular}

${ }^{*} \mathrm{p}<0.05$, SD: standard deviation; WHR: waist-hip ratio; BMI: body-mass index

fat percentage, fat mass, and body-mass index compared to the control group, suggesting that changes in body composition can be enhanced when near-infrared rays are irradiated into the abdomen during walking. The results of the present study support the previous studies that reported that near-infrared application had a significant effect on lipolysis ${ }^{9}$. In addition, the study by Ali et al. ${ }^{6}$ ) supports the results of the present study, reporting that obese adolescents showed a significant decrease in waist circumference, body fat percentage, and body-mass index compared to the control group when near-infrared rays were irradiated into the abdomen during aerobic exercise. The changes in body composition found in the present study may be due to the following: Several studies have shown that near-infrared light penetrates relatively deeply into the brain and tissues ${ }^{10)}$. Therefore, it is assumed that the near-infrared rays would be transmitted to the adipose tissue under the abdominal skin and generate heat, which would help break down adipose tissue. Hamblin and Demidova also support this study by claiming that when adipose tissue is irradiated with near-infrared rays, energy is generated to cause lipolysis ${ }^{11)}$.

Analysis of blood lipids (total cholesterol, HDL cholesterol, LDL cholesterol, triglyceride) showed no statistically significant difference between the experimental group and the control group. These results are consistent with the study of Mntrol et al. which found that there was no difference between an experimental group and a control group in total cholesterol, HDL cholesterol, LDL cholesterol, and triglycerides when obese women were treated with physical activity and near-infrared irradiation. However, though it is considered statistically insignificant in the scope of this relatively small study, a decrease in total cholesterol of $7.3 \mathrm{mg} / \mathrm{dl}$ and LDL cholesterol of $2.4 \mathrm{mg} / \mathrm{dl}$ in the experimental group after 4 weeks would seem to be meaningful figures ${ }^{12}$.

This study can be summarized as follows: The combination of near-infrared treatment and walking showed a positive effect on weight loss and abdominal fat loss. In general, adipose tissue is not easy to break down by exercise alone. In this respect, this study shows that irradiation of the body with near-infrared rays during walking can help to break down adipose tissue. The limitation of this study is that a possible placebo effect in the exercise group was not controlled. In future studies, investigation of multiple variables such as obesity levels, age groups, and sex in combination with various types of exercise is suggested.

\section{REFERENCES}

1) Jauch-Chara K, Oltmanns KM: Obesity—a neuropsychological disease? Systematic review and neuropsychological model. Prog Neurobiol, 2014,114 : 84-101. [Medline] [CrossRef]

2) Bray G, Saunders WB: Office management of obesity. Philadelphia: Saunders, 2003.

3) Matsuzawa Y, Shimomura I, Nakamura T, et al.: Pathophysiology and pathogenesis of visceral fat obesity. Diabetes Res Clin Pract, 1994, 24: S111-S116. [Medline] [CrossRef]

4) Caperchione CM, Vandelanotte C, Kolt GS, et al.: What a man wants: understanding the challenges and motivations to physical activity participation and healthy eating in middle-aged Australian men. Am J Men Health, 2012, 6: 453-461. [Medline] [CrossRef]

5) Manninen P, Riihimäki H, Heliövaara M, et al.: Overweight, gender and knee osteoarthritis. Int J Obes Relat Metab Disord, 1996, 20: 595-597. [Medline]

6) Ali A, Ryu KM, Kim SW, et al.: Effect of near infrared light application combined with aerobic exercise on excessive abdominal fat and obesity. Int J Adv Sci Tech, 2013, 61: 1-8. [CrossRef]

7) Mercer JB, Nielsen SP, Hoffmann G: Improvement of wound healing by water-filtered infrared-A (wIRA) in patients with chronic venous leg ulcers including evaluation using infrared thermography. GMS Ger Med Sci, 2008, 6 Doc 11. 
8) Hiatt WR, Wolfel EE, Meier RH, et al.: Superiority of treadmill walking exercise versus strength training for patients with peripheral arterial disease. Implications for the mechanism of the training response. Circulation, 1994, 90: 1866-1874. [Medline] [CrossRef]

9) Mordon SR, Wassmer B, Reynaud JP, et al.: Mathematical modeling of laser lipolysis. Biomed Eng Online, 2008, 7: 10. [Medline] [CrossRef]

10) Murkin JM, Arango M: Near-infrared spectroscopy as an index of brain and tissue oxygenation. Br J Anaesth, 2009, 103: i3-i13. [Medline] [CrossRef]

11) Hamblin MR, Demidova TN: Mechanisms of low level light therapy. In Biomedical Optics 2006. Int Soci Opti Photo, 2006. pp 614001-614001-12.

12) Möckel F, Hoffmann G, Obermüller R, et al.: Influence of water-filtered infrared-A (wIRA) on reduction of local fat and body weight by physical exercise. Ger Med Sci, 2006, 4: Doc05. [Medline] 